\title{
Correlation of Kupffer cell function and hepatocyte function in chronic viral hepatitis evaluated with superparamagnetic iron oxide-enhanced magnetic resonance imaging and scintigraphy using technetium-99m-labelled galactosyl human serum albumin
}

\author{
TATSUYUKI TONAN ${ }^{1}$, KIMINORI FUJIMOTO $^{1,4}$, ALIYA QAYYUM $^{8}$, SANAE AZUMA $^{1}$, MASATOSHI ISHIBASHI $^{1}$, \\ TAKATO UENO $^{3}$, NORIYUKI ONO ${ }^{6}$, JUNJI AKIYOSHI ${ }^{5}$, SUNAO MATSUSHITA ${ }^{7}$, \\ NAOFUMI HAYABUCHI ${ }^{1}$, TAKUMI KAWAGUCHI ${ }^{2}$ and MICHIO SATA ${ }^{2}$ \\ Departments of ${ }^{1}$ Radiology, ${ }^{2}$ Digestive Disease Information and Research, and ${ }^{3}$ Medicine, \\ Division of Cancer Center for Innovative Cancer Therapy, Kurume University School of Medicine; \\ ${ }^{4}$ Center for Diagnostic Imaging, Kurume University Hospital, Kurume 830-0011; ${ }^{5}$ Internal Medicine, \\ Kamitsu-Chuou Clinic, Kurume 830-0053; Departments of ${ }^{6}$ Internal Medicine, and ${ }^{7}$ Radiology, \\ Chikugo City Hospital, Chikugo 833-0041, Japan; ${ }^{8}$ Department of Radiology and Biomedical \\ Imaging, University of California-San Francisco, San Francisco, CA 94143, USA
}

Received January 20, 2011; Accepted April 1, 2011

DOI: 10.3892/etm.2011.255

\begin{abstract}
Kupffer cells contribute to the pathogenesis of liver injury in chronic liver disease, yet it is difficult to assess Kupffer cell function either ex vivo or in vivo, since supporting data are limited. The aim of this study was to clarify the relation between Kupffer cell function and hepatocyte function by analyzing the correlation between conventional indices of hepatic functional reserve and both superparamagnetic iron oxide-enhanced MRI (SPIO-MRI) and technetium-99mgalactosyl human serum albumin scintigraphy (Tc-99m-GSA) in patients with chronic viral hepatitis. Consecutive 46 patients
\end{abstract}

Correspondence to: Dr Kiminori Fujimoto, Department of Radiology, Kurume University School of Medicine, 67 Asahi-machi, Kurume 830-0011, Japan

E-mail:kimichan@med.kurume-u.ac.jp

Abbreviations: SPIO, superparamagnetic iron oxide; Tc-99mGSA-scintigraphy, scintigraphy using technetium-99m-labelled galactosyl human serum albumin; GRE, gradient-recalled echo; ROIs, regions of interest; \%LMR, percentages of liver-to-muscle signal intensity ratio; $\mathrm{H} 15$, uptake ratio of the heart at $15 \mathrm{~min}$ after Tc-99m-GSA injection; H3, uptake ratio of the heart at 3 min after Tc-99m-GSA injection; HH15, index of blood clearance $=\mathrm{H} 15 / \mathrm{H} 3$; L15, radioactivity of the liver at 15 min after Tc-99m-GSA injection; LHL15, the receptor index $=\mathrm{L} 15 /(\mathrm{H} 15+\mathrm{L} 15)$; ICG15, indocyanine green retention rate at $15 \mathrm{~min}$; $\mathrm{ROC}$ analysis, receiver operating characteristic analysis

Key words: chronic viral hepatitis, superparamagnetic iron oxide-enhanced magnetic resonance imaging, scintigraphy using technetium-99m-labelled galactosyl human serum albumin, Kupffer cell function, hepatic function reserve
(16 patients with chronic hepatitis and 30 patients with cirrhosis) who underwent both SPIO-MRI and Tc-99m-GSA were examined. The patients were aged 46-83 years (median 70) and included 29 men and 17 women. Spearman correlation coefficient was used to analyze the correlations between functional reserve indices and both reduction percentages of liver-to-muscle signal intensity ratio (reduction$\%$ LMR), as a surrogate parameter of Kupffer cell function and Tc-99m-GSA parameters. The usefulness of each parameter as a marker to differentiate Child-Pugh A from Child-Pugh B/C was evaluated using receiver operating characteristic (ROC) analysis. The reduction-\%LMR correlated more closely with Child-Pugh score $(\mathrm{r}=0.77 ; \mathrm{P}<0.001)$ than did Tc-99m-GSA parameters. For predicting Child-Pugh B/C, ROC analysis revealed that reduction-\%LMR $(\mathrm{AUC}=0.91, \mathrm{P}<0.001)$ was the most useful parameter and at a cutoff value of $50 \%$ or less, sensitivity, specificity, positive and negative predictive values were $0.79,0.91,0.94$ and 0.71 , respectively. SPIO-MRI may be a helpful non-invasive method for the evaluation of hepatic functional reserve, and this study suggests that Kupffer cell function is closely correlated with hepatocyte function in patients with chronic viral hepatitis.

\section{Introduction}

Proper assessment of hepatic functional reserve is crucial to the selection of patients for hepatic surgery, particularly in liver transplantation and the management of liver cirrhosis. The Child-Pugh score $(1,2)$ is now used worldwide as the standard in the assessment of hepatic functional reserve (3). Given the relation between this score and survival in patients with cirrhosis of the liver, it has recently been listed as one of the parameters in the minimal criteria of the liver transplant 
waiting list (4). The criteria are defined as a predicted 1-year survival rate of $\leq 90 \%$ (4), which generally includes those with a Child-Pugh score of $\geq 7$, i.e., Child-Pugh Class B or C.

A number of blood biochemical tests have been used to clinically evaluate hepatic functional reserve, including the serum albumin test, prothrombin time test, hepaplastin test, galactose-elimination capacity test (5), 14C-aminopyrine breath test (6), as well as testing for serum cholinesterase activity and indocyanine green retention rate at $15 \mathrm{~min}$ after administration (ICGR15) (7). Although these clinical parameters are indeed useful for assessing hepatic functional reserve, discrepancies between them and histological findings are common, suggesting in turn that the parameters do not always accurately reflect hepatic functional reserve $(8,9)$. Reserve function is often assessed by scintigraphy, particularly in Japan using technetium-99m-galactosyl human serum albumin (Tc-99m-GSA), a synthetic radioligand that binds to the asialoglycoprotein receptor on the plasma membrane of liver cells (10-12). Tc-99m-GSA scintigraphy enables more objective evaluation of hepatic functional reserve than the above clinical parameters (13). Many institutions have described methods for predicting hepatic functional reserve by Tc-99m-GSA scintigraphy (14-17). However, a consensus on the best method has not yet been reached (7).

Superparamagnetic iron oxide (SPIO) is a liver-specific particulate magnetic resonance (MR) contrast agent taken up by Kupffer cells (KCs) in the liver. Uptake of this agent reduces the signal-intensity of hepatic parenchyma and hepatic tumors, such as well-differentiated hepatocellular carcinoma (HCC) and dysplastic nodules (18) in accordance with the phagocytic activity of KCs. Previous studies have reported that cirrhotic tissue has a lower response to SPIO than noncirrhotic tissue due to the reduction of the phagocytic activity of KCs in a cirrhotic liver (19-23). Given the suspected close correlation between phagocytic activity of KCs and hepatic functional reserve, we speculated that SPIO-MRI may be suitable for the evaluation of hepatic functional reserve. However, it is difficult to assess KC function either ex vivo or in vivo, although morphologic evaluation of KCs can be estimated with immunohistologic staining and supporting data are limited.

To clarify the correlation between KC function and hepatocye function, we aimed to analyze the correlation between the conventional indices of hepatic functional reserve (biochemical markers and Child-Pugh score) and both SPIO-MRI and Tc-99m-GSA scintigraphy in patients with chronic viral hepatitis, and to determine whether or not SPIO-MRI effectively evaluates hepatic functional reserve.

\section{Materials and methods}

Patients. Our institutional review board approved our retrospective study and waived the informed consent requirement.

The inclusion criteria for the study were patients with chronic viral hepatitis who underwent SPIO-MRI for assessment of focal hepatic lesions and Tc-99m-GSA scintigraphy for assessment of hepatic functional reserve before hepatic surgery or interventional treatments for HCC. Consecutive 46 patients with hepatic dysfunction, who had hepatitis $C$ virus $(n=43)$ or hepatitis $B$ virus infections $(n=3)$ were enrolled in this retrospective study during a 6-month period.

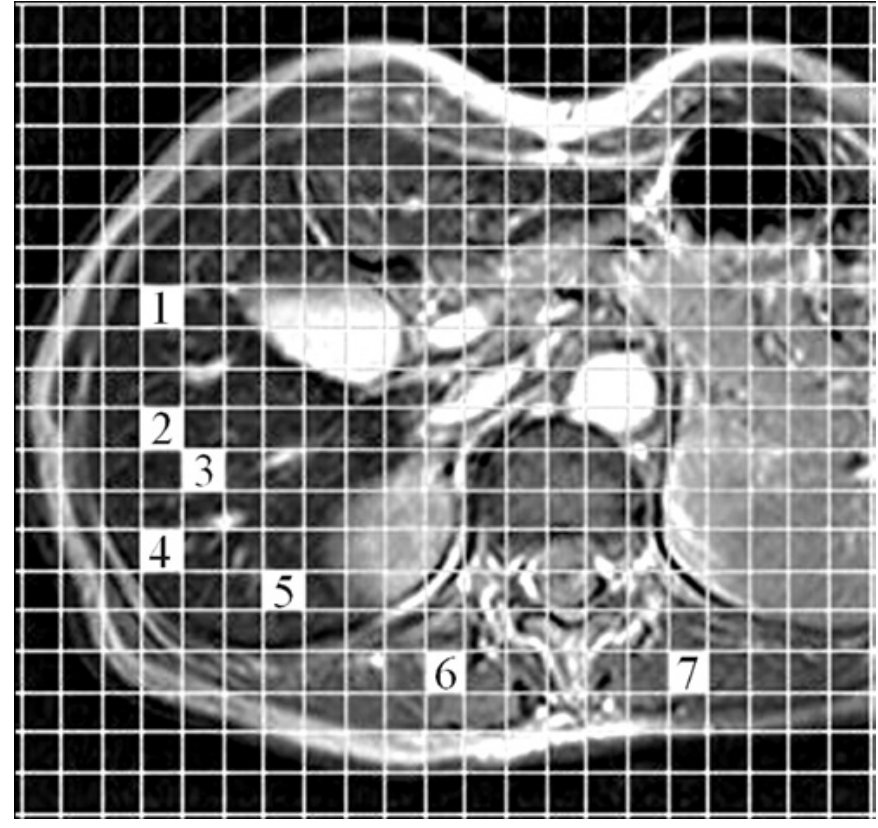

Figure 1. Measurement method of regions of interest (ROIs) on MRI. Using computer with the plug-in software, two independent observers freely and easily selected an ROI by clicking a mesh unit on an MR image for avoiding the large vessels, focal hepatic lesions or artifacts. One area shown on the MR image is $100 \mathrm{~mm}^{2}$. For liver parenchyma, 5 areas (1-5) were chosen and two areas (6 and 7) were chosen for paraspinous muscle.

There were no exclusions. The patients were aged 46-83 years (median 70) and included 29 men and 17 women. Twenty-nine patients were pathologically diagnosed by surgery for HCC or diagnostic hepatic biopsy under ultrasonography with a 22 -gauge core biopsy needle (cirrhosis, $n=22$; chronic hepatitis, $n=7$ ). The remaining 17 patients were diagnosed based on a range of imaging and clinical parameters, including findings on ultrasonography, computed tomography, endoscopic examination, biochemical markers and physical examination by two physicians (N.O. and J.A.) blinded to radiological image information.

The Child-Pugh score is generally categorized into three groups for evaluating hepatic functional reserve: class A, including patients with a score of 5 or 6 ; class B, including patients with a score from 7 to 9 ; and class $\mathrm{C}$, including patients with a score from 10 to 15 (2). In the present study, 46 patients were classified into four groups: chronic hepatitis (Child-Pugh score of $<5$; group $\mathrm{CH}, \mathrm{n}=16$ ); cirrhosis with Child-Pugh class A (Child-Pugh A, $\mathrm{n}=11$ ); and Child-Pugh class $B(n=16)$ or $C(n=3)$ (hereafter, Child-Pugh $B / C, n=19)$.

Superparamagnetic iron oxide-magnetic resonance imaging (MRI). MRI was performed at a field strength of 1.0-T (Gyroscan T10-NT; Philips, Best, Netherlands) using a body phased-array coil. Ferumoxides (Feridex; Eiken Chemical, Tokyo, Japan) were administered as SPIO via slow intravenous drip infusion (median $30 \mathrm{~min}$; range 25-36) at a dose of $10 \mu \mathrm{mol} / \mathrm{kg}$. MRI was performed $30 \mathrm{~min}$ after completion of the infusion.

T2-weighted gradient-recalled echo (GRE) images were obtained in the transverse plane from all patients before and after administration of SPIO. The scanning parameters on the 

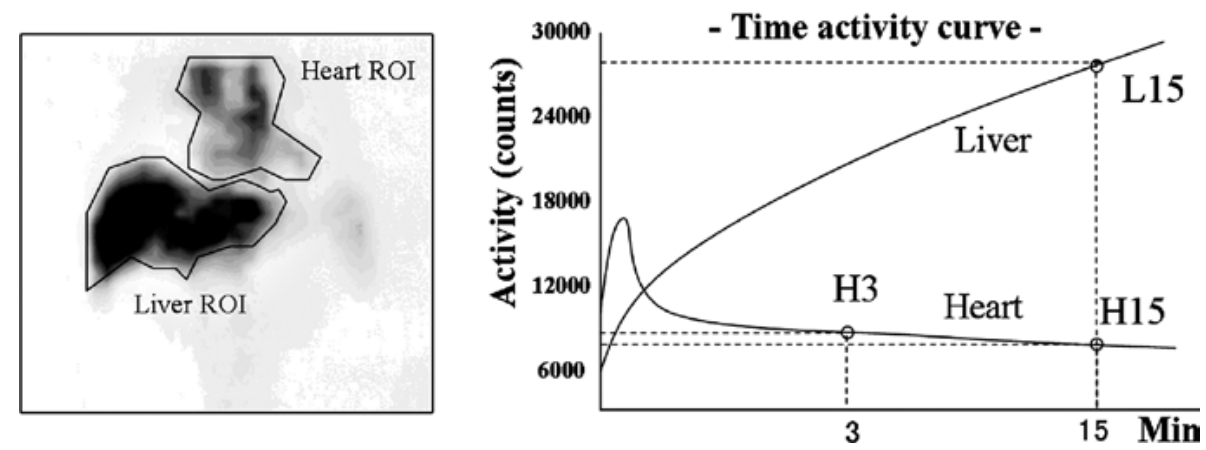

Figure 2. Time-activity curves for the heart and liver were generated from regions of interest (ROIs) for the whole liver and precordium. Index of blood clearance $(\mathrm{HH} 15=\mathrm{H15} / \mathrm{H} 3)$ and receptor index $(\mathrm{LHL} 15=\mathrm{L} 15 / \mathrm{H} 15+\mathrm{L} 15)$ were calculated by the radioactivity of liver and heart ROIs.

T2-weighted GRE sequence were as follows: repetition time $=280 \mathrm{msec}$, echo time $=14 \mathrm{msec}$, flip angle $=30^{\circ}$, an $8-\mathrm{mm}$ section thickness, a 1-mm gap, a $35-\mathrm{cm}$ field of view, $256 \mathrm{x}$ 205 rectangular matrix, 16 sections and one signal acquired during a 22 -sec breath hold. These scanning parameters were unchanged for T2-weighted GRE sequences before and after administration of SPIO.

Quantitative image analysis was conducted by measurement of signal intensities of the liver parenchyma and paraspinous muscle. All regions of interest (ROIs) were determined by two independent radiologists (T.T. and A.S.) using plug-in software developed in-house by one of the authors (K.F.) (Fig. 1). Five separate ROIs were placed over the right hepatic lobe on the T2-weighted GRE images in three different slice sections, and liver signal intensities were recorded as the mean values generated (liver ROI area, 500 $\mathrm{mm}^{2}$ ). Liver signal intensities were measured before and after administration of SPIO, avoiding large vessels, focal hepatic lesions or artifacts in the same locations on the images. The procedure was repeated to measure muscle signal intensity by placing two separate ROIs on the right and left paraspinous muscles, avoiding the intermuscular fat on the transverse sections used to measure liver signal intensity (muscle ROI area, $200 \mathrm{~mm}^{2}$ ). Liver-to-muscle signal intensity ratio (LMR) was calculated for T2-weighted GRE images before and after administration of SPIO (24).

To assess the effect of SPIO for each patient group, the reduction percentage of LMR (reduction-\%LMR) before and after administration of SPIO was calculated as follows:

LMR = mean signal intensities of the liver parenchyma/ mean signal intensities of the muscle.

Reduction-\%LMR $(\%)=($ post-LMR - pre-LMR)/pre-LMR $\mathrm{x} 100$, where pre-LMR is the LMR before SPIO injection and post-LMR is the LMR after injection.

Tc-99m galactosyl human serum albumin scintigraphy. All parameters for Tc-99m-GSA scintigraphy were assessed by consensus of two observers (M.I. and S.M.) independently of the previously mentioned observers. All patients underwent hepatic imaging with Tc-99m-GSA under a $\gamma$-camera (ZLC-7500; Shimadzu, Kyoto, Japan) placed over the chest abdominal field. After an overnight fast, $185 \mathrm{MBq}$ of Tc-99m-GSA was administered by bolus injection. The patient was then placed in a supine position and a dynamic image was captured. Anterior abdominal images, including the heart and liver, were obtained continuously for $30 \mathrm{~min}$. Time-activity curves for the heart and liver were generated from ROIs covering the whole organ.

Quantitative indices were calculated from the time-activity curves, including the index of blood clearance $(\mathrm{HH} 15=\mathrm{H} 15 /$ $\mathrm{H} 3$ ), which is defined as the uptake ratio of the heart at $15 \mathrm{~min}$ (H15) to that at $3 \mathrm{~min}(\mathrm{H} 3)$ after Tc-99m-GSA injection, and the receptor index $(\mathrm{LHL} 15=\mathrm{L} 15 / \mathrm{H} 15+\mathrm{L} 15)$, which is defined as the uptake ratio of the liver at $15 \mathrm{~min}$ to that of the liver and heart at 15 min after injection, where L15 is the radioactivity of the liver at $15 \mathrm{~min}$ after injection (Fig. 2). The index of blood clearance was calculated by dividing the radioactivity of the heart ROI at 15 min after Tc-99m-GSA injection by that of the heart ROI at $3 \mathrm{~min}$ after injection (25). The receptor index was calculated by dividing the radioactivity of the liver ROI by that of the liver plus heart ROIs at 15 min after the injection (25).

The median interval between SPIO-MRI and Tc-99m-GSA scintigraphy was 10 days (range 2-18).

Statistical analysis. Skewed data were summarized for all non-parametric methods using the median and the 25 th to 75th percentile of the interquartile range.

A Bland-Altman plot (26) was used to analyze inter-observer agreement for the reduction-\%LMR on T2-weighted GRE images. The correlation of the reduction-\%LMR on T2-weighted GRE images obtained by the two observers was determined using the Pearson correlation coefficient. Spearman's rank correlation test was used to analyze the association between the conventional indices of hepatic functional reserve (biochemical markers and Child-Pugh score) with the reduction-\%LMR on SPIO-MRI as well as each Tc-99m-GSA scintigraphy parameter (i.e., HH15 or LHL15). The Kruskal-Wallis test was used to determine the significance of intergroup differences between reduction-\%LMR, HH15 and LHL15. When results revealed statistical significance, multiple comparisons of each coupled combination were conducted using the Mann-Whitney U test with Bonferroni correction.

In the cirrhotic groups, receiver operating characteristic (ROC) analysis was performed to evaluate the usefulness of reduction-\%LMR, HH15 or LHL15 as markers for differentiating Child-Pugh A from Child-Pugh $\mathrm{B} / \mathrm{C}$ when selecting liver transplant patients from a waiting list (4). Calculations of area under the ROC curve (AUC) found a range from 0.5 to 1.0 , which increased when diagnostic performance 


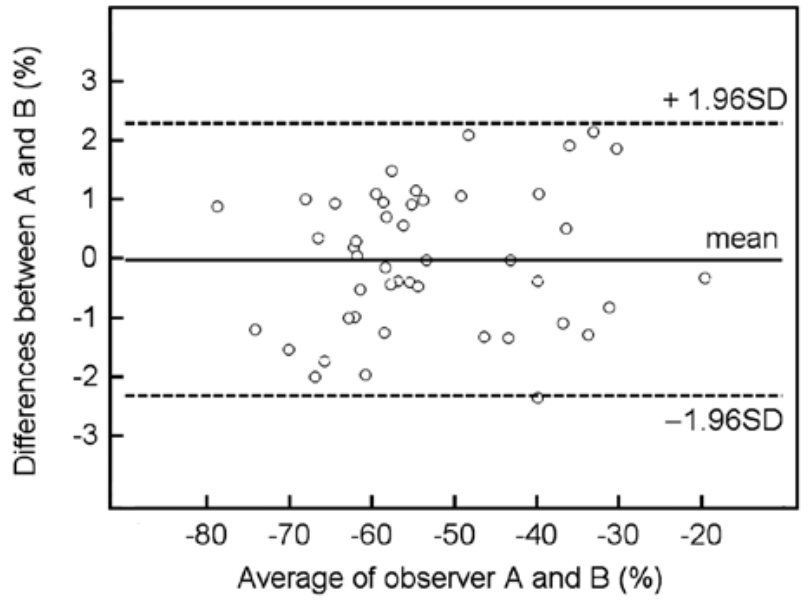

Figure 3. Graph (Bland-Altman plot) displays a scatter diagram of the differences plotted against the average of the two independent observers' measurements of the reduction-\%LMR on T2-weighted GRE image. Horizontal solid line is drawn at the mean difference and horizontal dotted lines are drawn at the mean differences \pm 1.96 times the standard deviation (SD) of the differences.

approached that of the reference standard (in the present case, determination of Child-Pugh B/C). Sensitivity, specificity, positive predictive value and negative predictive value were calculated with standard formulas according to the values of these indices and with varied index values that indicated positive differentiation (i.e., threshold value).

$\mathrm{P}<0.05$ was considered statistically significant. All analyses were performed using SPSS statistical software (version 12.0 J; SPSS, Inc., Chicago, IL, USA).

\section{Results}

Interobserver agreement for the reduction-\%LMR on T2-weighted GRE images. There was no significant difference between measurements made by the two observers; the interclass Pearson correlation coefficient was $0.98(95 \%$ confidence interval, 0.97-0.99) for reduction-\%LMR; the mean difference ( \pm standard deviation) was $-0.018 \pm 1.17 \%$ and the coefficient repeatability was 2.29. The Bland-Altman plot with $95 \%$ limits of agreement is shown in Fig. 3. There was no proportional bias or fixed bias.

Correlation between the conventional indices (biochemical markers and Child-Pugh score) and parameters of each modality. Results showed the reduction-\%LMR to be positively correlated with asparatate aminotransferase (Spearman $\mathrm{r}=0.52 ; \mathrm{P}<0.001)$, ICGR15 ( $\mathrm{r}=0.58 ; \mathrm{P}<0.001)$, hyaluronic acid $(\mathrm{r}=0.53 ; \mathrm{P}<0.001)$ and Child-Pugh score $(\mathrm{r}=0.77 ; \mathrm{P}<0.001)$. The reduction-\%LMR was found to be negatively correlated with serum albumin level $(r=-0.62$; $\mathrm{P}<0.001)$, platelet count $(\mathrm{r}=-0.55 ; \mathrm{P}<0.001)$ and prothrombin time $(\mathrm{r}=-0.56 ; \mathrm{P}<0.001)$. Correlations between the conventional indices of hepatic functional reserve and reduction-\%LMR, HH15 and LHL15 are shown in Table I.

Significant intergroup differences for the parameters of each modality. Significant differences were found between the four groups for reduction-\%LMR, HH15 and LHL15
Table I. Correlation of the parameters on SPIO-MRI and Tc-99m-GSA scintigraphy with conventional indices.

\begin{tabular}{|c|c|c|c|}
\hline & SPIO-MRI & \multicolumn{2}{|c|}{$\begin{array}{l}\text { Tc-99m-GSA } \\
\text { scintigraphy }\end{array}$} \\
\hline & Reduction-\%LMR & HH15 & LHL15 \\
\hline AST (IU/l) & $0.52^{\mathrm{a}}$ & $0.59^{\mathrm{a}}$ & $-0.62^{\mathrm{a}}$ \\
\hline ALT (IU/l) & 0.19 & 0.19 & -0.21 \\
\hline$\gamma$-GTP (IU/l) & 0.10 & 0.24 & -0.18 \\
\hline T-Bil (mg/dl) & $0.36^{\mathrm{b}}$ & $0.57^{\mathrm{a}}$ & $-0.50^{\mathrm{a}}$ \\
\hline $\mathrm{Alb}(\mathrm{g} / \mathrm{dl})$ & $-0.62^{\mathrm{a}}$ & $-0.61^{\mathrm{a}}$ & $0.64^{\mathrm{a}}$ \\
\hline Plt $\left(10^{4} / \mu \mathrm{l}\right)$ & $-0.55^{\mathrm{a}}$ & $-0.64^{\mathrm{a}}$ & $0.69^{\mathrm{a}}$ \\
\hline PT (\%) & $-0.56^{\mathrm{a}}$ & $-0.47^{b}$ & $0.49^{b}$ \\
\hline ICGR15 (\%) & $0.58^{\mathrm{a}}$ & $0.67^{\mathrm{a}}$ & $-0.73^{a}$ \\
\hline HA (ng/ml) & $0.53^{\mathrm{a}}$ & $0.74^{\mathrm{a}}$ & $-0.73^{a}$ \\
\hline Child-Pugh score & $0.77^{\mathrm{a}}$ & $0.62^{\mathrm{a}}$ & $0.60^{\mathrm{a}}$ \\
\hline
\end{tabular}

Reduction-\%LMR, reduction percentages of liver-to-muscle signal intensity ratio; HH15, index of blood clearance; LHL15, receptor index; AST, glutamic-oxaloacetic transaminase; ALT, alanine aminotransferase; r-GTP, r-glutamyl transpeptidase; T-Bil, total-bilirubin; Alb, serum albumin level; Plt, platelet count; PT, prothrombin time; ICG15, indocyanine green retention rate at $15 \mathrm{~min}$; HA, hyaluronic acid. Values are Spearman's rank correlation coefficient (Spearman r). ${ }^{\mathrm{a}} \mathrm{P}<0.001 .{ }^{\mathrm{b}} \mathrm{P}<0.01$.

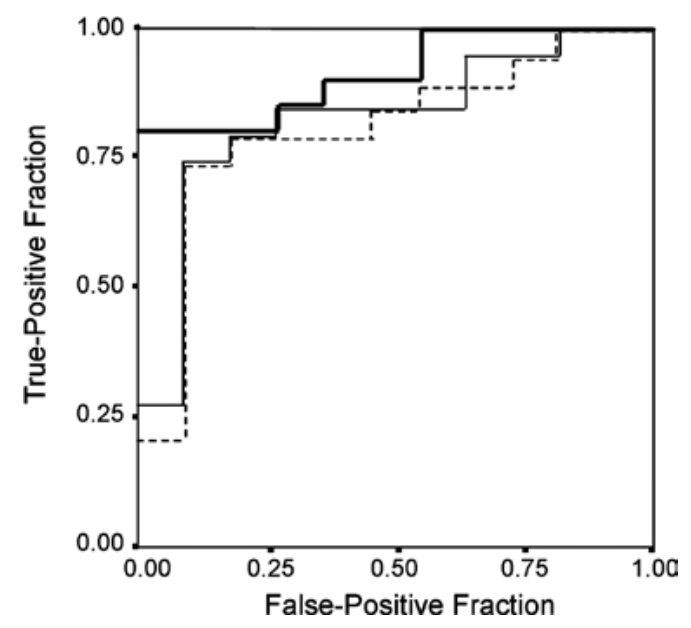

Figure 4. Graphs show receiver operating characteristic curve for differentiating Child-Pugh class B/C from Child-Pugh class A. Note fraction of true-positive results (sensitivity) and false-positive results (1 minus specificity) for reduction-\%LMR (thick solid line), LHL15 (thin solid line) and HH15 (dotted line). Value of 0.5 is no better than expected by chance and value of 1.0 reflects perfect indicator. Calculated area under curve is 0.91 for reduction-\%LMR, 0.82 for LHL15 and 0.81 for $\mathrm{HH} 15$.

(Kruskal-Wallis, $\mathrm{P}<0.001$, all comparisons). With regard to each coupled combination, significant differences in reduction-\%LMR and HH15 were found between group $\mathrm{CH}$ and Child-Pugh $\mathrm{B}$, between group $\mathrm{CH}$ and Child-Pugh $\mathrm{C}$, between Child-Pugh A and Child-Pugh B, and between Child-Pugh A and Child-Pugh $\mathrm{C}$ (Bonferroni test, $\mathrm{P}<0.05$, all comparisons), but no difference was found between group $\mathrm{CH}$ and Child-Pugh A or between Child-Pugh B and Child-Pugh C 
Table II. Multiple comparisons of each group coupled combination for reduction-\%LMR, HH15 and LHL15.

\begin{tabular}{|c|c|c|c|}
\hline \multirow[b]{2}{*}{$\begin{array}{l}\text { Group according to hepatic } \\
\text { function reserve }\end{array}$} & \multirow{2}{*}{$\frac{\text { SPIO-MRI }}{\text { Reduction-\%LMR }}$} & \multicolumn{2}{|c|}{ Tc-99m-GSA scintigraphy } \\
\hline & & HH15 & LHL15 \\
\hline $\mathrm{CH}(\mathrm{n}=16)$ & $-62.1(-67.7,-58.6)^{\mathrm{a}}$ & $0.51(0.59,0.43)^{\mathrm{a}}$ & $0.92(0.94,0.88)^{\mathrm{a}}$ \\
\hline Class A $(n=11)$ & $-57.7(-62.0,-54.7)^{\mathrm{a}}$ & $0.58(0.63,0.51)^{\mathrm{b}}$ & $0.88(-0.91,0.84)^{\mathrm{b}}$ \\
\hline Class B $(n=16)$ & $-41.5(-53.2,-36.1)^{\mathrm{a}}$ & $0.75(0.78,0.61)^{\mathrm{a}, \mathrm{b}}$ & $0.78(0.84,0.75)^{\mathrm{a}, \mathrm{b}}$ \\
\hline Class C $(n=3)$ & $-33.7(-39.9,-25.0)^{\mathrm{a}}$ & $0.85(0.85,0.84)^{\mathrm{a}, \mathrm{b}}$ & $0.62(0.65,0.49)^{\mathrm{a}, \mathrm{b}}$ \\
\hline
\end{tabular}

$\mathrm{CH}$, patients with chronic hepatitis; Class A, Child-Pugh score 5 or 6; Class B, Child-Pugh score 7 to 9; Class C, Child-Pugh score 10 to 15 . Values are the medians of reduction percentages of liver-to-muscle signal intensity ratio (reduction-\%LMR), the index of blood clearance (HH15) and the receptor index (LHL15). Values in parentheses are the 25th and 75th percentile of interquartile ranges. P-values are corrected by Bonferroni method after calculating by using the Mann-Whitney $\mathrm{U}$ test; ${ }^{\mathrm{a}} \mathrm{P}<0.001,{ }^{\mathrm{b}} \mathrm{P}<0.05$.

Table III. Diagnostic rates of reduction-\%LMR according to cutoff value for differentiation of Child-Pugh class A and class $\mathrm{B} / \mathrm{C}$.

\begin{tabular}{|c|c|c|c|c|c|c|}
\hline \multirow[b]{2}{*}{ Variable $^{\mathrm{a}}$} & \multicolumn{6}{|c|}{ Diagnostic rate (95\% confidence interval) } \\
\hline & $\begin{array}{l}\text { Liver cirrhosis } \\
\qquad(\mathrm{n}=30)\end{array}$ & P-value ${ }^{b}$ & Sensitivity & Specificity & PPV & NPV \\
\hline \multicolumn{7}{|c|}{ Reduction-\%LMR } \\
\hline$<-50 \%$ & 16 & $<0.001$ & $0.79(0.54-0.94)$ & $0.91(0.59-0.99)$ & $0.94(0.70-0.99)$ & $0.71(0.42-0.92)$ \\
\hline$\geq 50 \%$ & 14 & & & & & \\
\hline
\end{tabular}

PPV, positive predictive value; NPV, negative predictive value for determination of malignancy. ${ }^{a} \mathrm{~A}$ receiver operating characteristic curve was used to select the best cutoff points. ${ }^{b}$ Based on results of generalized estimating equation analysis of the association between the severity of liver cirrhosis and values of reduction percentages of liver-to-muscle signal intensity ratio (reduction-\%LMR). If the reduction-\%LMR is less than $-50 \%$, the liver cirrhosis is considered Child-Pugh class B or C.

(Table II). For LHL15, in addition to the results mentioned above, a significant difference was found between Child-Pugh B and Child-Pugh C (Table II).

ROC analysis for the parameters of each modality in cirrhotic groups. Reduction-\%LMR [AUC $=0.91 ; 95 \%$ confidence interval (CI) $0.80-1.00, \mathrm{P}<0.001]$ was a more useful parameter in differentiating Child-Pugh A from Child-Pugh $\mathrm{B} / \mathrm{C}$ than HH15 (AUC $=0.80 ; 95 \%$ CI $0.64-0.98$ ) or LHL15 (AUC $=0.82$; 95\% CI 0.66-0.98) (Fig. 4). ROC analysis indicated that the $-50 \%$ threshold level for reduction-\%LMR was suitable for differentiating Child-Pugh A from Child-Pugh $\mathrm{B} / \mathrm{C}$. Based on the threshold values of $-50 \%$, the sensitivity, specificity, positive predictive and negative predictive values for reduction-\%LMR were $0.79,0.91,0.94$ and 0.71 , respectively (Table III).

\section{Discussion}

In the present study, we demonstrated that SPIO-MRI was a useful non-invasive method for evaluating hepatic functional reserve. Furthermore, reduction-\%LMR, as a surrogate parameter of phagocytic activity of KCs, was found to be well correlated with Child-Pugh score and also with biochemical

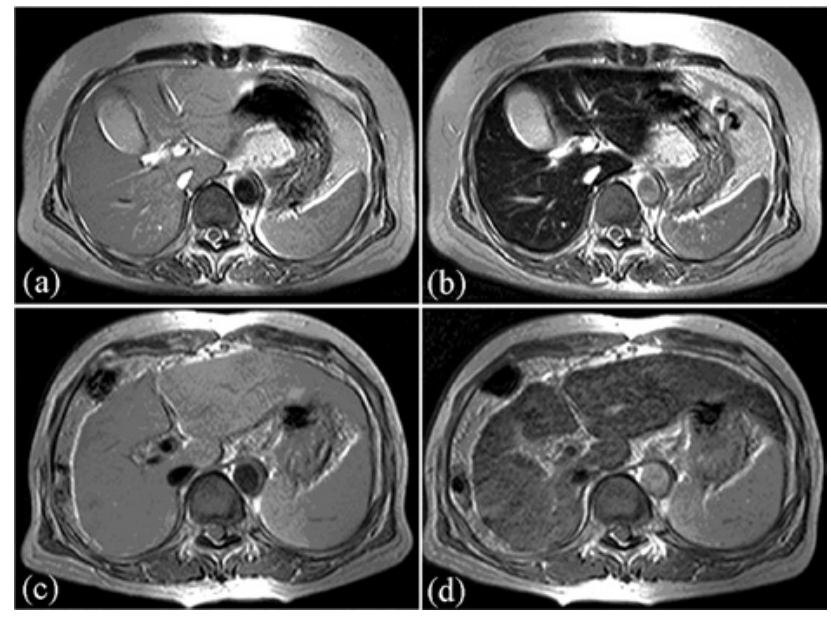

Figure 5. MR images on T2-weighted GRE sequence in a 51-year-old woman with chronic hepatitis (A and B) and a 65-year-old woman with liver cirrhosis (C and D) (Child-Pugh class B, score 8). Before (A and C) and after (B and D) administration of SPIO. Signal intensity reduction of liver parenchyma in a patient with cirrhosis (reduction-\%LMR, 38.2\%) is less than chronic hepatitis (reduction-\%LMR, 73.8\%).

markers, particularly serum albumin, prothrombin time and ICGR15 in patients with chronic viral hepatitis (Table I). 
These findings support the hypothesis that phagocytic activity of KCs is closely correlated with hepatocyte function in patients with chronic viral hepatitis $(27,28)$.

Pathologically, hepatic cirrhosis is defined as an irreversible diffuse fibrosis or scarring of the liver. Cirrhosis causes a decrease in hepatic function reserve and is a common endpoint for many chronic liver diseases. Previous studies have reported that the decrease in signal intensity in SPIOproduced liver parenchyma is not as great in patients without cirrhosis as in those with cirrhosis (19-23) (Fig. 5).

SPIO-produced signal intensity is dependent on both the number of sinusoidal KCs per unit volume of hepatic parenchyma (KC density) and the level of activity in each KC (22). Namely, it has been considered to represent the phagocytic activity of KCs. However, recent studies have reported that impairment of $\mathrm{KC}$ phagocytic activity is dependent on the level of activity in each $\mathrm{KC}$ and is not dependent on a reduction in the total number of KCs in studies of animal models of non-alcoholic steatohepatitis $(29,30)$.

Reduction-\%LMR on SPIO-MRI may, therefore, reflect the level of activity in each $\mathrm{KC}$ in chronic viral hepatitis. In other words, the level of activity in each KC may be closely correlated with hepatocyte function.

With regard to the evaluation of hepatic functional reserve by biochemical markers, several authors have reported that markers, such as serum albumin level and prothrombin time, do not accurately reflect hepatic functional reserve, since these parameters are affected by the blood product supplements used to treat liver cirrhosis. Furthermore, these studies also reported that the ICGR15 does not allow for reserve measurement in patients with elevated bilirubin levels and portosystemic shunts $(11,12)$.

On the other hand, several methods have been reported for calculating hepatic Tc-99m-GSA uptake in order to ensure maximum clinical efficacy in the evaluation of reserve by Tc-99m-GSA scintigraphy (10-12). Previous studies reported that Tc-99m-GSA scintigraphy is able to evaluate the decrease in hepatic functional reserve caused by portosystemic shunts more precisely than biochemical markers $(31,32)$.

In the present study, there were significant differences among groups for HH15 and LHL15 (Table II). We also found that these parameters were well correlated with the Child-Pugh score and with biochemical markers, as well as with the results of previous studies on Tc-99m-GSA scintigraphy $(10-12,25)$. However, in comparison to SPIO-MRI with scintigraphy, our results showed that Child-Pugh score and prothrombin time are closely correlated with reduction\%LMR on SPIO-MRI rather than HH15 and LHL15 on Tc-99m-GSA scintigraphy.

Several authors have investigated the efficacy of SPIO-MRI for the detection of hepatic fibrosis in patients with chronic liver disease $(33,34)$; their results showed that SPIO-MRI was useful for evaluating hepatic fibrosis. In the present study, there was statistical correlation between SPIO-MRI and hyaluronic acid, which is used widely as a marker of hepatic fibrosis $(35,36)$. This result also supports that reduction-\%LMR on SPIO-MRI may be useful for evaluating hepatic fibrosis, potentially allowing for more accurate evaluation of hepatic functional reserve.
Liver transplantation is now firmly established as a general treatment for patients with terminal liver disease. Generally, the minimal criteria for liver transplantation are those established by the American Society of Transplant Physicians and American Association for the Study of Liver Disease in 1997, regardless of etiology (4). The criteria are defined as a predicted 1-year survival rate of $\leq 90 \%$ (4), which generally includes those with a Child-Pugh class B/C. In the present study, ROC curve analysis showed that reduction-\%LMR was a more useful parameter in differentiating Child-Pugh A from Child-Pugh B/C than HH15 or LHL15 (Fig. 4). Furthermore, the cutoff value of less than $-50 \%$ of reduction-\%LMR indicated high specificity and was suitable for diagnosing Child-Pugh $\mathrm{B} / \mathrm{C}$ in patients with cirrhosis (specificity 0.91 and positive predictive value 0.94) (Table III). Therefore, SPIO-MRI may be useful to predict early cirrhosis in patients with chronic viral hepatitis and may be a suitable technique which supports evaluation of hepatic functional reserve by Child-Pugh score in the selection of liver transplant patients from a waiting list.

Several limitations of the present study warrant mention. First, the study was conducted under a retrospective design and the sample size was small. Second, some bias may have arisen from patient selection, since histopathological diagnosis was performed in 29 of the 46 patients, whereas the remaining 17 were clinically diagnosed by two physicians. Third, the T2-weighted GRE images in the present study were designed for a 1.0-T MRI unit.

In conclusion, SPIO-MRI may be a useful non-invasive method suitable for the evaluation of hepatic functional reserve, suggesting that $\mathrm{KC}$ phagocytic activity is closely correlated with hepatocyte function in patients with chronic viral hepatitis.

\section{References}

1. Child CG and Turcotte JG: Surgery and portal hypertension. Major Probl Clin Surg 1: 1-85, 1964.

2. Pugh RNH, Murray-Lyon IM, Dawson JL, Pietroni MC and Williams R: Transection of the oesophagus for bleeding oesophageal varices. Br J Surg 60: 646-649, 1973.

3. Schneider PD: Preoperative assessment of liver function. Surg Clin North Am 84: 355-373, 2004.

4. Lucey MR, Brown KA, Everson GT, et al: Minimal criteria for placement of adults on the liver transplant waiting list. Transplantation 66: 956-962, 1998.

5. Tygstrup $\mathrm{N}$ : The galactose elimination capacity in control subjects and in patients with cirrhosis of the liver. Acta Med Scand 175: 281-289, 1964

6. Hepner GW and Vesell ES: Assessment of aminopyrine metabolism in man by breath analysis after oral administration of 14C-aminopyrine. N Engl J Med 291: 134-147, 1975.

7. Kawamura H, Kamiyama T, Nakagawa T, et al: Preoperative evaluation of hepatic functional reserve by converted ICGR15 calculated from Tc-GSA scintigraphy. J Gastroenterol Hepatol 8: 1235-1241, 2008.

8. Moody FG, Rikkers LF and Aldrete JS: Estimation of the functional reserve of human liver. Ann Surg 180: 592-598, 1972.

9. Yamanaka N, Okamoto E, Kuwata K and Tanaka N: A multiple regression equation for prediction of posthepatectomy liver failure. Ann Surg 200: 658-663, 1984.

10. Fujioka H, Kawashita Y, Kamohara Y, et al: Utility of technetium-99m-labeled-galactosyl human serum albumin scintigraphy for estimating the hepatic functional reserve. J Clin Gastroenterol 28: 329-333, 1999. 
11. Sasaki N, Shiomi S, Iwata Y, et al: Clinical usefulness of scintigraphy with $99 \mathrm{mTc}$-galactosyl-human serum albumin for prognosis of cirrhosis of the liver. J Nucl Med 40: 1652-1656, 1999.

12. Kawamura E, Shiomi S, Ishizu H, et al: Natural course of change in hepatic functional reserve in patients with chronic liver disease evaluated by scintigraphy with GSA. Hepatol Res 27: 129-135, 2003

13. Shiomi S, Kuroki T, Kuriyama M, et al: Evaluation of fluminant hepatic failure by scintigraphy with technetium-99m-GSA. J Nucl Med 38: 79-82, 1997.

14. Hakawa SK and Tanaka Y: A quantitative model of technetium99m-DTPA-galactosyl-HSA for the assessment of hepatic blood flow and hepatic binding receptor. J Nucl Med 32: 2233-2240, 1991.

15. Sugai Y, Komatani A, Hosoya T and Takahashi K: Analysis of the early blood kinetics of $99 \mathrm{mTc}-\mathrm{GSA}$ and its verification: new one-compartment model and regression equation. Nucl Med Commun 22: 773-778, 2001.

16. Sugahara K, Togashi H, Takahashi K, et al: Separate analysis of asialoglycoprotein receptors in the right and left hepatic lobes using Tc-GSA SPECT. Hepatology 38: 1401-1409, 2003.

17. Nanashima A, Yamaguchi H, Shibasaki S, et al: Relationship between indocyanine green test and technetium-99m galactosyl serum albumin scintigraphy in patients scheduled for hepatectomy: clinical evaluation and patient outcome. Hepatol Res 28: 184-190, 2004

18. Tonan T, Fujimoto K, Azuma S, et al: Evaluation of small $(<$ or $=2 \mathrm{~cm}$ ) dysplastic nodules and well-differentiated hepatocellular carcinomas with ferucarbotran-enhanced MRI in a 1.0-T MRI unit: utility of T2-weighted gradient echo sequences with an intermediate-echo time. Eur J Radiol 64: 133-139, 2007.

19. Elizondo G, Weissleder R, Stark DD, et al: Hepatic cirrhosis and hepatitis: MR imaging enhanced with superparamagnetic iron oxide. Radiology 174: 797-801, 1990.

20. Clement O, Frija G, Chambon C, et al: Liver tumors in cirrhosis: experimental study with SPIO-enhanced MR imaging. Radiology 180: 31-36, 1991.

21. Kato N, Ihara S, Tsujimoto T and Miyazawa T: Effect of resovist on rats with different severities of liver cirrhosis. Invest Radiol 222: 661-666, 2002.

22. Tanimoto A, Yuasa Y, Shinmoto H, et al: Superparamagnetic iron oxide-mediated hepatic signal intensity change in patients with and without cirrhosis: pulse sequence effects and Kupffer cell function. Radiology 222: 661-666, 2002.

23. Hundt W, Petsch R, Heimberger T and Reiser H: Signal changes in liver and spleen after Endorem administration in patients with and without liver cirrhosis. Eur Radiol 10: 409-416, 2000.
24. Gandon Y, Olivié D, Guyader D, et al: Non-invasive assessment of hepatic iron stores by MRI. Lancet 31: 357-362, 2004.

25. Kudo M, Todo A, Ikekubo K, et al: Functional hepatic imaging with receptor-binding radiopharmaceutical: clinical potential as a measure of functioning hepatocyte mass. Gastroenterol Jpn 26: 734-741, 1991.

26. Bland JM and Altman DG: Statistical methods for assessing agreement between two methods of clinical measurement. Lancet 1: 307-310, 1986.

27. Waxman AD: Scintigraphic evaluation of diffuse hepatic disease. Semin Nucl Med 12: 75-88, 1982.

28. Klingensmith WC III, Fritzberg AR, Zerbe GO and Koep LJ: Relative role of Tc-99m-diethyl-IDA and Tc-99m-sulfur colloid in the evaluation of liver function. Clin Nucl Med 5: 341-346, 1980.

29. Asanuma T, Ono M, Kubota K, et al: Super paramagnetic iron oxide MRI shows defective Kupffer cell uptake function in nonalcoholic fatty liver disease. Gut 59: 258-266, 2010.

30. Tsujimoto T, Kawaratani H, Kitazawa T, et al: Decreased phagocytic activity of Kupffer cells in a rat nonalcoholic steatohepatitis model. World J Gastroenterol 14: 6036-6043, 2008.

31. Kira T, Tomiguchi S, Kira M, Ohyama Y and Takahashi M: Quantitative evaluation of the hepatic functional reserve using technetium-99m DTPA-galactosyl human serum albumin before and after transjugular intrahepatic portosystemic shunt. Eur J Nucl Med 24: 1268-1272, 1997.

32. Osada H, Honda N, Takahashi T, et al: Relationship between (99m)Tc-GSA scintigraphic indices of liver function reserve and portal circulation in patients with chronic liver disease. Ann Nucl Med 21: 245-249, 2007.

33. Aguirre DA, Behling CA, Alpert E, Hassanein TI and Sirlin CB: Liver fibrosis: noninvasive diagnosis with double contrast material-enhanced MR imaging. Radiology 239: 425-437, 2006.

34. Lucidarme O, Baleston F, Cadi M, et al: Non-invasive detection of liver fibrosis: Is superparamagnetic iron oxide particleenhanced MR imaging a contributive technique? Eur Radiol 13: 467-474, 2003

35. Engström-Laurent A, Laurent UB, Lilja K and Laurent TC: Concentration of sodium hyaluronate in serum. Scand J Clin Lab Invest 45: 497-504, 1985.

36. Tsukamoto T, Yamamoto T, Ikebe T, et al: Serum markers of liver fibrosis and histologic severity of fibrosis in resected liver. Hepatogastroenterology 51: 777-780, 2004. 